\title{
Analysis of adnexal mass managed during cesarean section
}

\author{
Cheng $Y_{u^{* 1,2, B-D}}$, Jie Wang ${ }^{* 1, B-D}$, Weiguo $\mathrm{Lu}^{1, C, E}$, Xing Xie ${ }^{1, A, E}$, Xiaodong Cheng ${ }^{1, B, C}$, Xiao $\mathrm{Li}^{1, A, B, B, F}$ \\ ${ }^{1}$ Women's Hospital, Zhejiang University School of Medicine, Hangzhou, China \\ 2 Hangzhou Women's Hospital, China \\ A - research concept and design; $B$ - collection and/or assembly of data; $C$ - data analysis and interpretation; \\ $D$ - writing the article; $E$ - critical revision of the article; $F$ - final approval of the article
}

\section{Address for correspondence \\ Xiao Li \\ E-mail:5198008@zju.edu.cn \\ * Cheng Yu and Jie Wang contributed equally to this article.}

\section{Funding sources}

This study was funded by the projects of Zhejiang Province Natural Scientific Foundation for Distinguished Young Scientists (grant No. LR15H160001) and by Foundation of Science and Technology Department of Zhejiang Province, China (grant No. 2012(13019-3).

\section{Conflict of interest}

None declared

Received on February 22, 2017

Reviewed on May 4, 2017

Accepted on September 20, 2017

Published online on August 7, 2018

\begin{abstract}
Background. Pregnancy with an adnexal mass is one of the most common complications during pregnancy and clinicians are sometimes caught in a dilemma concerning the decision to be made regarding clinical management.
\end{abstract}

Objectives. The objective of this study was to outline and discuss the clinical features, management and outcomes of adnexal masses that were encountered during a cesarean section (CS) at a university-affiliated hospital in China.

Material and methods. The medical records of the patients with an adnexal mass observed during a CS were retrospectively collected at Women's Hospital, Zhejiang University School of Medicine, Hangzhou, China, from January 1991 to December 2011.

Results. The incidence of adnexal masses was 16.40 per 1000 CSs. The most common pathologic diagnosis was benign ovarian tumor, the $2^{\text {nd }}$ was ovarian endometrioma and the $3^{\text {rd }}$ was theca lutein cyst. Thirteen cases of ovarian malignancies were diagnosed during a CS. Only 388 cases (29.78\%) were detected using an ultrasonography (USG) examination before a CS. Eight cases required emergency CS due to abdominal pain; all other patients were clinically asymptomatic. The reasons for abdominal pain included torsion ( $n=5)$, rupture $(n=2)$ and ovarian enlargement $(n=1)$. In 13 cases with ovarian endometrioma, cysts ruptured during a CS without any clinical manifestation. No maternal and fetal complications related to surgery were observed.

Conclusions. Preconception care and routine prenatal care, including USG examination, may optimize the detection and management of an adnexal mass. The presumptive ovarian endometrioma detected before pregnancy could be the indication for surgery due to the possibility of spontaneous hemoperitoneum. Theca lutein cysts might be huge and exist throughout the whole pregnancy period. Expectant management is reasonable for an adnexal mass that emerged during pregnancy without suspicion of malignancy. Abdominal pain might be a clue for cyst torsion or rupture.

Key words: pregnancy, cesarean delivery, adnexal mass

\section{Cite as}

Yu C, Wang J, Lu W, Xie X, Cheng X, Li X. Analysis of adnexal mass managed during cesarean section. Adv Clin Exp Med. 2019;28(4):447-452. doi:10.17219/acem/77099

DOI

10.17219/acem/77099

\section{Copyright}

Copyright by Author(s)

This is an article distributed under the terms of the

Creative Commons Attribution Non-Commercial License

(http://creativecommons.org/licenses/by-nc-nd/4.0/) 


\section{Introduction}

Pregnancy with an adnexal mass is one of the most common complications during pregnancy, with an incidence of $1-5.3 \%$, according to previous reports. ${ }^{1-5}$ Improper management of an adnexal mass during pregnancy can endanger the maternal and neonatal safety, owing to the particularity of the pregnancy. Both the fetus and mother should be taken into account when the doctors try to manage the adnexal mass. Due to the development of ultrasonography (USG) technology and the awareness regarding prenatal health care, an increased number of adnexal masses are detected during pregnancy. ${ }^{6,7}$ As we know, the risk of malignancy of adnexal masses during pregnancy cannot be determined by an USG examination alone, and the true pathologic type can be determined only by surgical examination of the masses. Thus, the clinician sometimes would be caught in a dilemma concerning whether to choose surgery or expectant management. Moreover, despite the widespread use of USG technology, the incidence of adnexal masses incidentally discovered during cesarean section (CS) remains high. This would potentially carry risk for clinical management. The aim of the present study was to compare and report the clinical characteristics of adnexal masses with different pathologic types that were encountered during CS at Women's Hospital, Zhejiang University School of Medicine, Hangzhou, China. It is also the intention of this study to provide a reference for diagnosis and treatment of adnexal masses during pregnancy.

\section{Material and methods}

This is a clinical retrospective study of gravid women with an adnexal mass diagnosed during CS at our hospital in the 21-year period between January 1991 and December 2011. Approval from the Ethics Committee of the hospital was obtained before the study was conducted. Owing to the retrospective character of the study, informed consent was not needed. The following data was collected: patient's age, gestational age, gravidity and parity history, symptoms, USG records before CS, indication for CS, the size and site of the adnexal mass at CS, surgical procedure, pathologic diagnosis, perioperative complications, and neonatal outcome. Final diagnosis was confirmed by a routine paraffin section after surgery. The pathology specialists at our hospital reviewed all pathologic sections. The present conditions of the patients with malignancy were followed up and the information was retrospectively recorded up to the date of the last contact. For theca lutein cysts, the manifestations were followed up until the masses dissolved. Data was analyzed using the $\chi^{2}$ test. Statistical analysis was performed with SPSS v. 20.0 software (IBM Corp., Armonk, USA). The p-values below 0.05 were considered statistically significant.

\section{Results}

\section{Basic information}

There were 79,548 CSs at our hospital during the studied 21 years. Briefly, 1,303 cases of adnexal masses were diagnosed during CS. The incidence was 1.64\%. Among patients undergoing CS, the median age was 29 years (20-42 years). The median pregnancy duration was 37 weeks (28-42 weeks), including 1,263 (96.93\%) women with a duration $\geq 34$ weeks and $40(3.07 \%)$ women with a duration <34 weeks; 1,215 (93.25\%) women were primipara and 88 women were multipara (6.75\%). All women underwent CS for different indications (Table 1). Of the

Table 1. The indications for cesarean section (CS)

\begin{tabular}{|l|c|c|}
\hline \multicolumn{1}{|c|}{ Indication } & $\mathrm{n}[\%]$ & Indication \\
\hline Social factor & $238(18.27)$ & cord entanglement \\
\hline Fetal distress & $150(11.51)$ & placenta previa/abruption \\
\hline Adnexal mass & $148(11.36)$ & multiple pregnancy \\
\hline Abnormal fetal position & $147(11.28)$ & IVF-ET \\
\hline Cephalopelvic disproportion/arrested labor ${ }^{1}$ & $124(9.52)$ & medical disease ${ }^{2}$ \\
\hline Oligohydramnion & $88(6.75)$ & surgical diseases ${ }^{3}$ \\
\hline Intrahepatic cholestasis of pregnancy & $60(4.60)$ & s.46) \\
\hline High myopia & $54(2.07)$ & 26 (2.00) \\
\hline Scar uterus ${ }^{4}$ & $45(3.45)$ & postterm pregnancy \\
\hline Preeclampsia & $45(3.46)$ & induced labor failure \\
\hline
\end{tabular}

IVF-ET - in vitro fertilization and embryo transfer; ${ }^{1}$ cephalopelvic disproportion/arrested labor: macrosomia, uterine inertia, cervix edema, pendulous abdomen, protracted active phase, contracted pelvic outlet, prolonged latent phase; ${ }^{2}$ medical diseases: cardiac disease, kidney disease, hematological system disease, gestational hypertension, gestational diabetes mellitus, pregnancy-induced eczema; ${ }^{3}$ surgical diseases: uterine malformation, tail bone fracture, varicosity, pendulous abdomen, lumbar disc herniation; ${ }^{4}$ scar uterus: 41 cases were previous cesarean deliveries and 4 cases had history of uterine surgery; categorical variables were expressed as number and percentage. 
1,303 patients, $1,290(99.00 \%)$ cases received a prenatal visit. Only 388 (29.78\%) masses were detected by a USG examination before surgery; 132 (34.02\%) masses were found before pregnancy, 120 masses (30.93\%) in the $1^{\text {st }}$ trimester, 63 masses $(16.24 \%)$ in the $2^{\text {nd }}$ trimester, and 73 masses $(18.81 \%)$ in the $3^{\text {rd }}$ trimester. No sign of malignancy was observed for these masses during pregnancy. The remaining 915 (70.22\%) cases were incidentally discovered during CS.

\section{Clinical manifestations}

Most masses were clinically asymptomatic, except for 8 (0.61\%) cases which required emergency CS due to acute abdominal pain. The reasons for abdominal pain included torsion ( $\mathrm{n}=5$, 3 for benign ovarian tumor, 1 for ovarian endometrioma and 1 for theca lutein cyst), rupture ( $n=2$;
1 for benign ovarian tumor and 1 for ovarian endometrioma) and ovarian enlargement ( $\mathrm{n}=1$; theca lutein cyst) (Table 2). In 13 patients with ovarian endometrioma, the cyst ruptured during CS without any obvious abdominal pain and discomfort during pregnancy. Among them, 4 masses were detected before pregnancy, 3 cases in the $1^{\text {st }}$ trimester and 6 cases at CS. Among 179 cases with theca lutein cyst, only 19 cases were secondary to assisted reproductive technologies.

\section{Diagnosis and management}

A frozen section was applied for suspected malignant mass. The final diagnosis was made according to the pathologic report by the paraffin section. The clinical characterstics and management are presented in Table 3 and the pathologic types of adnexal masses are shown

Table 2. Cases that received emergency cesarean section (CS) due to acute abdominal pain

\begin{tabular}{|c|c|c|c|c|c|}
\hline Pathology & GA [weeks] & Complication & Management & Lateral & Size $[\mathrm{cm}]$ \\
\hline Mature teratoma & 39 & torsion & cystectomy & $R$ & 9 \\
\hline Serous cystadenoma & 37 & torsion & cystectomy & $\mathrm{R}$ & 7 \\
\hline Mucinous cystadenoma & 37 & torsion & SO & $\mathrm{R}$ & 15 \\
\hline Mucinous cystadenoma & 38 & rupture & so & $L$ & 30 \\
\hline Ovarian endometrioma & 28 & torsion & cystectomy & $\mathrm{Bi}$ & 4,4 \\
\hline Ovarian endometrioma & 35 & rupture & cystectomy & $\mathrm{R}$ & 6 \\
\hline Theca lutein cyst & 38 & torsion & so & $L$ & 5 \\
\hline Theca lutein cyst & 38 & NA & so & $\mathrm{Bi}$ & 9,10 \\
\hline
\end{tabular}

GA - gestational age; SO - salpingo-oophorectomy; R - right; L - left; Bi - bilateral; NA - not applicable.

Table 3. Clinical characteristics and management of 1,303 adnexal masses

\begin{tabular}{|c|c|c|c|c|c|c|c|c|}
\hline Pathology & $\mathrm{n}[\%]$ & Cystectomy & SO & Uni & $\mathrm{Bi}$ & $<5 \mathrm{~cm}$ & $5 \mathrm{~cm}$ & $>5 \mathrm{~cm}$ \\
\hline \multicolumn{9}{|c|}{ Benign ovarian tumor } \\
\hline Mature teratoma & 390 (29.93) & 380 & 10 & 353 & 37 & 140 & 84 & 166 \\
\hline Serous cystadenoma & 155 (11.90) & 153 & 2 & 149 & 6 & 109 & 19 & 27 \\
\hline Mucinous cystadenoma & $76(5.83)$ & 62 & 14 & 75 & 1 & 18 & 9 & 49 \\
\hline Fibroma & $26(2.00)$ & 25 & 1 & 26 & 0 & 23 & 1 & 2 \\
\hline Mixed cystadenoma & $8(0.61)$ & 8 & 0 & 7 & 1 & 4 & 3 & 1 \\
\hline Ovarian leiomyoma & $1(0.08)$ & 1 & 0 & 1 & 0 & 1 & 0 & 0 \\
\hline Brenner tumor & $1(0.08)$ & 1 & 0 & 1 & 0 & 0 & 1 & 0 \\
\hline Theca cell tumor & $1(0.08)$ & 1 & 0 & 1 & 0 & 0 & 0 & 1 \\
\hline Ovarian endometrioma & $251(19.26)$ & 243 & 8 & 219 & 32 & 168 & 32 & 51 \\
\hline \multicolumn{9}{|c|}{ Ovarian tumor-like condition } \\
\hline Theca lutein cyst* & 179 (13.74) & 74 & 2 & 73 & 106 & 54 & 27 & 98 \\
\hline Simple cyst & $103(7.90)$ & 103 & 0 & 101 & 2 & 73 & 12 & 18 \\
\hline Stromal hyperplasia & $34(2.61)$ & 34 & 0 & 34 & 0 & 34 & 0 & 0 \\
\hline Paraovarian-paratubal cyst & $65(4.99)$ & 65 & 0 & 64 & 1 & 50 & 6 & 9 \\
\hline Ovarian malignancy** & $13(1.00)$ & 3 & 9 & 13 & 0 & 6 & 2 & 5 \\
\hline
\end{tabular}

SO - salpingo-oophorectomy; Uni - unilateral; Bi - bilateral; * 103 theca lutein cysts were not listed in this table, they received biopsy (40), paracentesis (2) or were untreated (61); ** 1 patient with ovarian carcinoma, who received radical surgery, was not listed here (the detailed data of ovarian malignancy was published in Reference 8); categorical variables were expressed as number and percentage. 


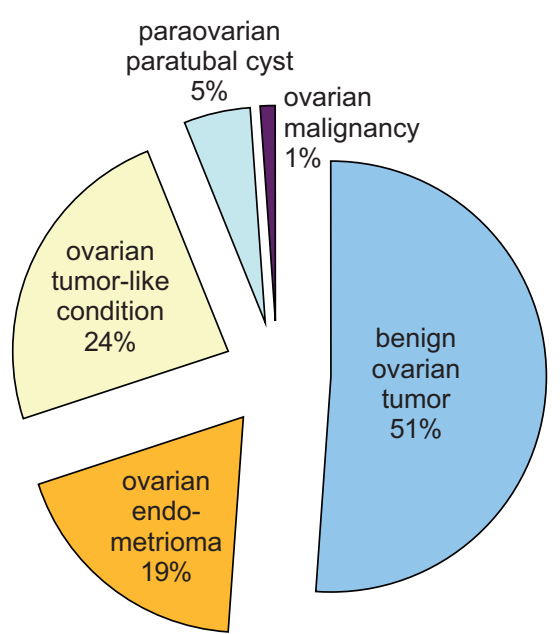

Fig. 1. The pathological types distribution of 1,303 adnexal masses

in Fig 1. In brief, the most common histological type was mature teratoma $(390,29.93 \%)$, the $2^{\text {nd }}$ was ovarian endometrioma $(251,19.26 \%)$, the $3^{\text {rd }}$ was theca lutein cyst $(179,13.74 \%)$, and the $4^{\text {th }}$ was serous cystadenoma $(155$, $11.90 \%) ; 13(1.00 \%)$ were ovarian malignancies. ${ }^{8}$ The median adnexal mass size for benign ovarian tumor was $5 \mathrm{~cm}$ (range from 0.1 to $30 \mathrm{~cm}$ ), $6 \mathrm{~cm}$ for theca lutein cyst (from 0.3 to $16 \mathrm{~cm}$ ), $4 \mathrm{~cm}$ for ovarian endometrioma (from 0.5 to $13 \mathrm{~cm}$ ), $3 \mathrm{~cm}$ for paraovarian-paratubal cyst (from 0.5 to $10 \mathrm{~cm}$ ), and $5 \mathrm{~cm}$ for ovarian malignancy (from 2.5 to $30 \mathrm{~cm}$ ). The frequencies of unilateral and bilateral of the adnexal mass were 1,117 (85.73\%) and 186 (14.27\%), respectively. After excluding the rare pathologic types, we found that most masses were unilateral (84.80\%), except for theca lutein cysts (40.80\%); the differences were significant $\left(\chi^{2}=330.799, p=0.000\right)$. Most masses had the size of $5 \mathrm{~cm}$ or less $(65.70 \%)$, except for theca lutein cysts (45.30\%); the differences were significant $\left(\chi^{2}=142.134, p=0.000\right)$. A cystectomy was performed in $1,153(88.49 \%)$ cases. Unilateral salpingo-oophorectomy (SO) was performed in 45 (3.53\%) cases. Bilateral $\mathrm{SO}$ was performed in 1 case with theca lutein cyst due to misdiagnosis as malignancy. One ovarian carcinoma received radical surgery, including total abdominal hysterectomy, $\mathrm{SO}$ and pelvic lymphadenectomy during CS. Briefly, 103 cases with theca lutein cysts were treated with biopsy (40), paracentesis (2) or were untreated (61). All the 103 theca lutein cysts resolved spontaneously within 1-12 months after CS, which was confirmed by an USG examination, and no complications, such as cyst torsion or rupture, presented during the follow-up period. A total of 1,338 healthy live newborns were recorded, including 178 premature newborns (28-37 weeks). No fetal mortality and congenital malformations were detected in the newborns. No complications, morbidity and mortality related to surgical removal of the masses during CS were observed. No tumor metastasis to placenta and fetus was recorded for ovarian malignancies at the mean follow-up time of 108.7 months. All patients with malignancies are currently in complete remission and tumor-free.

\section{Discussion}

Depending on the method one uses and how one defines a clinically significant adnexal mass, the prevalence of pregnancies complicated by an adnexal mass varies. ${ }^{1-3}$ In accordance with previous reports, we found that the incidence of adnexal masses diagnosed during CS was about $1.64 \%$. As we know, a USG examination is the most helpful method in the detection and evaluation of an adnexal mass in women, regardless of whether the woman is pregnant or not. Moreover, it can detect masses smaller than those usually detected during a physical examination, especially during pregnancy. ${ }^{3}$ Aggarwal and Kehoe reviewed the studies conducted between 1984 and 2009 and found that a USG examination increased the detection of adnexal masses in pregnancies from $0.04-1.32 \%(1 / 76-1 / 2,328)$ to $1.14-5.23 \%(1 / 19-1 / 88) .{ }^{6}$ The majority of masses found during pregnancies are small $(<5 \mathrm{~cm})$ and represent functional cysts that typically resolve spontaneously by the $2^{\text {nd }}$ trimester. ${ }^{1}$ Our data reflects the actual rate of adnexal masses in CS patients, which can explain why the rate is situated in the low limitation of the previous reports. Nevertheless, the incidence of masses discovered during CS was much higher than that $(0.49 \%)$ detected before and during pregnancy in the present study. Only $29.78 \%$ of the adnexal masses were detected before CS, although about $99.00 \%$ gravida women received a prenatal examination. This means that more than half of the masses were diagnosed incidentally during CS. This is similar to the results of a study by Baser et al., which revealed that $83(55.0 \%)$ adnexal masses were incidentally discovered during CS. ${ }^{7}$ The probable reasons for this low diagnostic rate during pregnancy were addressed here. Most adnexal masses were asymptomatic and small $(\leq 5 \mathrm{~cm})$; the pregnant patients refused a pelvic examination and a transvaginal USG examination for the fear of abortion during early pregnancy; some adnexal masses might emerge after pregnancy and gravid uterus may obscure the correct visualization and detection. Thus, it is important to promote the use of USG and improve the USG technique during preconception and prenatal visits in China.

Once the adnexal mass is detected during pregnancy, the obstetrician would have to make the clinical decision of how to manage the mass, since the masses can affect the pregnancy outcome due to the risk for torsion, rupture, bleeding, obstruction, or malignancy. Even today, the management of adnexal masses during pregnancy is controversial. ${ }^{9-10}$ While some obstetricians prefer elective removal in the $2^{\text {nd }}$ trimester, others state that a conservative approach results in the resolution of most masses and avoid unnecessary surgery. ${ }^{6}$ Surgery during pregnancy carries some inherent intraoperative and perioperative risks, including the added risks of fetal loss, preterm contractions and an increased risk of embolic events. ${ }^{1,11}$ However, observing a mass during pregnancy might delay the treatment if the adnexal mass is malignant or develops an acute 
event, such as ovarian torsion, cyst rupture or obstruction of labor, which often necessitates emergency intervention. ${ }^{1}$ The pathologic types in the present study are concordant with those in previous reports. ${ }^{7,12-14}$ Owing to their characteristic USG appearance, the differential diagnosis for most masses is relatively easy with the improvement of the USG technique, especially for an experienced sonographer. However, some malignancies were still misdiagnosed before CSs in our study and in the reports from other researchers. The rate of malignancy in the present study was $1.00 \%$ (13/1303), including 8 low-malignant potential tumors, 3 invasive epithelial carcinomas and 2 malignant germ cell tumors. ${ }^{8}$ All malignancies were clinically asymptomatic and were diagnosed in stage I with good prognosis. Thus, we agree that delaying surgery may be feasible to avoid any unnecessary risks to the pregnancy, depending on the clinical suspicion of malignancy. ${ }^{1}$ For asymptomatic adnexal masses, surgery should only be considered in a pregnancy for suspicious or obvious malignant tumors. ${ }^{15}$ No new malignances were diagnosed after 2008 in the present study. This might be due to the improved technique of USG and most suspicious adnexal masses receiving surgery before CS. Nevertheless, routine puerperium USG is strongly recommended for vaginal delivery, since it raises the possibility for delaying the diagnosis of an adnexal mass.

In the present study, we found that 20 (1.61\%) cases experienced torsion or rupture. A previous study reported that ovarian torsion was most commonly (60\% of the time) seen in pregnant women with an adnexal mass. ${ }^{1}$ Interestingly, $75 \%$ of complications were connected to ruptures and most of them in the present study were ovarian endometriomas. However, the rate of torsion would be increased to $71.43 \%(5 / 7)$ if the asymptomatic ruptures of ovarian endometrioma were excluded. The complications managed during early or mid-pregnancy were excluded, because we only collected the information of the patients with an adnexal mass observed during CS; as a result, the actual rate of torsion or rupture here was lower than in previous reports. During the last decade, the increased use of assisted reproductive technologies has led to higher fertility rates in patients with ovarian endometrioma. Therefore, the number of pregnant women with ovarian endometrioma and associated complications may rise, despite most investigators reporting regression or cessation of growth of the endometriomas during pregnancy. ${ }^{4,16,17}$ Theoretically, ovarian endometrioma torsion and rupture may be less common during pregnancy due to its adhesion to the neighboring tissues, and pregnancy may have a beneficial effect on endometriosis by promoting involution of decidualizing ectopic endometrium. ${ }^{18-20}$ However, we found that $71.43 \%(15 / 21)$ of the masses with complications were ovarian endometriomas and most of them (14/15) were ruptures. This is concordant with a previous study, which reports that ovarian endometrioma is a major risk factor for spontaneous hemoperitoneum in pregnancy. ${ }^{18}$ Fortunately, no massive hemoperitoneum presented in this study. Of the 15 cases, only 2 received emergency CS due to acute abdomen pain. Other 13 cases with ruptures were asymptomatic and diagnosed at the time of the CS for other surgical indications. Although ovarian endometrioma is widely studied, little is known about the incidence of ovarian endometrioma complications during pregnancy. This study is the first detailed report on the rupture and torsion in ovarian endometrioma observed during a relatively large number of CSs. The possibility of spontaneous hemoperitoneum should be always kept in mind, since $7.97 \%$ of ovarian endometriomas experienced a rupture in the present study. ${ }^{17}$

Functional cysts usually resolve spontaneously by the $2^{\text {nd }}$ trimester, while we found that 179 (13.74\%) were theca lutein cysts, which persisted until CS. Theca lutein cysts, also known as hyperreactio luteinalis (HL), are a type of functional ovarian cyst. ${ }^{21,22}$ They are typically multiple and bilateral, and are usually associated with gestational trophoblastic disease, ovulation induction and very rarely with a normal pregnancy. Interestingly, only 19 cases received ovulation induction in the present study. Production of high concentrations of human chorionic gonadotropin (HCG) and increased ovarian sensitivity to prolonged exposure to HCG may be manifested as an exaggerated ovarian response, leading to theca lutein cyst formation. ${ }^{23,24}$ In the present study, 1 case with HL was misdiagnosed as a malignancy during CS and received bilateral SO (Table 2). The bilateral masses were $10 \mathrm{~cm}$ in size and the surgeon did not recognize them. After that, all obstetricians in our hospital were alerted to the possibility that huge theca lutein cysts can exist through the whole trimester and mimic malignancy. That is why doctors should be aware that theca lutein cysts may mimic malignancies and lead to an unnecessary ovarian resection. ${ }^{21,25}$ Furthermore, most theca lutein cysts are bilateral (54.7\%), which might be helpful for a differential diagnosis. In the present study, most theca lutein cysts were asymptomatic and were found incidentally with USG or during CS; $34.08 \%$ of cases were managed expectantly by experienced obstetricians and showed to resolve spontaneously postpartum. Thus, the management should be carefully chosen depending on the manifestation of the masses. It is important to exclude malignancies with a biopsy (or wedge resection) and then freeze the section in order to avoid unnecessary surgical excision. ${ }^{21}$ Paracentesis is acceptable for huge masses to minimize their volume and avoid complications if no malignancy signs are present, while a cystectomy and SO are not recommended unless there is a definite indication.

\section{Conclusions}

Based on our results and previous reports, an USG examination is still the preferred auxiliary examination to rule out the adnexal mass, although most masses in the present study were incidentally discovered during CS. Preconception care, routine prenatal care and puerperal checks, 
including USG examinations, may be used to optimize the detection and management of adnexal masses, since most masses are unilateral and $\leq 5 \mathrm{~cm}$. Active treatment is advised for the persistent adnexal masses or presumptive ovarian endometriomas found before pregnancy, to avoid acute abdominal disease or other complications related to pregnancy that could threaten maternal-fetal safety. For the masses detected during pregnancy, expectant management is recommended if no malignancy is suspected and closer observation is recommended. ${ }^{6,26}$ The presumed risk of torsion or rupture should not be considered as an indication for surgery. ${ }^{27}$ Moreover, the patients should also be aware that antenatal surgery might become necessary once the mass becomes symptomatic or its features change. ${ }^{6}$ For the masses discovered during CS, surgical removal is preferred to prevent subsequent complications (torsion or rupture) or a future requirement for surgery, except for functional cysts such as theca lutein cysts. ${ }^{6,28,29}$

\section{References}

1. Horowitz NS. Management of adnexal masses in pregnancy. Clin Obstet Gynecol. 2011;54(4):519-527.

2. Naqvi M, Kaimal A. Adnexal masses in pregnancy. Clin Obstet Gynecol. 2015;58(1):93-101.

3. Bernhard L, Klebba P, Gray DL, Mutch D. Predictors of persistence of adnexal masses in pregnancy. Obstet Gynecol. 1999;93(4):585-589.

4. Reif P, Schöll W, Klaritsch P, Lang U. Rupture of endometriotic ovarian cyst causes acute hemoperitoneum in twin pregnancy. Fertil Steril. 2011;95(6):2125.e1-e3.

5. Gholson R, Pullen J, Miles A, Gardner MO, Doyle NM. Adnexal masses in pregnancy: Does magnitude matter? Obstet Gynecol. 2014;123: 192S-193S.

6. Aggarwal $P$, Kehoe S. Ovarian tumours in pregnancy: A literature review. Eur J Obstet Gynecol Reprod Biol. 2011;155(2):119-124.

7. Baser E, Erkilinc S, Esin S, et al. Adnexal masses encountered during cesarean delivery. Int J Gynaecol Obstet. 2013:123(2):124-126.

8. LiX, Yang X. Ovarian malignancies incidentally diagnosed during cesarean section: Analysis of 13 cases. Am J Med Sci. 2011;341(3):181-184.

9. Surampudi K, Nirmalan PK, Gundabattula SR, Chandran JB. Management of adnexal masses in pregnancy: Our experience from a tertiary referral perinatal centre in south India. Arch Gynecol Obstet. 2015;291(1):53-58.

10. Ngu SF, Cheung VY, Pun TC. Surgical management of adnexal masses in pregnancy. JSLS. 2014;18(1):71-75.
11. Kim $\mathrm{H}$, Jenkins TR. Evaluation and management of adnexal mass in pregnancy. Am J Obstet Gynecol. 2011;205(2):97-102.

12. Yacobozzi M, Nguyen D, Rakita D. Adnexal masses in pregnancy. Semin Ultrasound CT MR. 2012;33(1):55-64.

13. Goh W, Bohrer J, Zalud I. Management of the adnexal mass in pregnancy. Curr Opin Obstet Gynecol. 2014;26(2):49-53.

14. Türkçüoğlu I, Meydanli MM, Engin-Ustün Y, Ustün Y, Kafkasli A. Evaluation of histopathological features and pregnancy outcomes of pregnancy associated adnexal masses. J Obstet Gynaecol. 2009;29(2): 107-109.

15. Marret $H$, Lhommé $C$, Lecuru F, et al. Guidelines for the management of ovarian cancer during pregnancy. Eur J Obstet Gynecol Reprod Biol. 2010;149(1):18-21.

16. Barri PN, Coroleu B, Tur R, Barri-Soldevila PN, Rodriguez I. Endometriosis-associated infertility: Surgery and IVF, a comprehensive therapeutic approach. Reprod Biomed Online. 2010;21(2):179-185.

17. Ueda $Y$, Enomoto T, Miyatake T, et al. A retrospective analysis of ovarian endometriosis during pregnancy. Fertil Steril. 2010;94(1):78-84.

18. Brosens IA, Fusi L, Brosens JJ. Endometriosis is a risk factor for spontaneous hemoperitoneum during pregnancy. Fertil Steril. 2009;92(4): 1243-1245.

19. Brosens JJ, Parker MG, McIndoe A, Pijnenborg R, Brosens IA. A role for menstruation in preconditioning the uterus for successful pregnancy. Am J Obstet Gynecol. 2009;200(6):164-168.

20. Brosens JJ, Gellersen B. Death or survival: Progesterone-dependent cell fate decisions in the human endometrial stroma. J Mol Endocrinol. 2006;36(3):389-398.

21. Upadhyaya G, Goswami A, Babu S. Bilateral theca lutein cysts: A rare cause of acute abdomen in pregnancy. Emerg Med Australas. 2004;16(5-6): 476-477.

22. Onodera N, Kishi I, Tamaoka Y, Yamazaki K, Kamei K. A case of recurrent hyperreactio luteinalis. Am J Obstet Gynecol. 2008;198(5):e9-e10.

23. Amoah C, Yassin A, Cockayne E, Bird A. Hyperreactio luteinalis in pregnancy. Fertil Steril. 2011;95(7):2429.e1-e3.

24. Li Q, Li X, Zhang P. Ovarian torsion caused by hyperreactio luteinalis in the third trimester of pregnancy: A case report. Int J Clin Exp Med. 2015;8(10):19612-19615.

25. Atis A, Cifci F, Aydin Y, Ozdemir G, Goker N. Hyperreactio luteinalis with preeclampsia. J Emerg Trauma Shock. 2010;3(3):298.

26. Koo FH, Wang KC, Chen CY, et al. An 11-year experience with ovarian surgery during pregnancy. J Chin Med Assoc. 2013;76:452-457.

27. Bottomley C, Bourne T. Diagnosis and management of ovarian cyst accidents. Best Pract Res Clin Obstet Gynaecol. 2009;23(5):711-724.

28. Behtash N, Karimi Zarchi M, Modares Gilani M, Ghaemmaghami F, Mousavi A, Ghotbizadeh F. Ovarian carcinoma associated with pregnancy: A clinicopathologic analysis of 23 cases and review of the literature. BMC Pregnancy Childbirth. 2008;8:3.

29. Machado $F$, Vegas $C$, Leon J, et al. Ovarian cancer during pregnancy: Analysis of 15 cases. Gynecol Oncol. 2007;105(2):446-450. 